\title{
The Results of Deep CCD Field Surveys: Very Low Mass Halo Population Stars As Dark Matter
}

\author{
P. C. Boeshaar \\ Drew University, Madison, NJ 07940 \\ boeshaa@drew.drew.edu \\ J. A. Tyson \\ AT\&T Bell Laboratories, Murray Hill, NJ 07974 \\ tyson@physics.att.com \\ G. M. Bernstein \\ University of Michigan, Ann Arbor, MI 48109 \\ garyb@astro.lsa.umich.edu
}

\begin{abstract}
Halo and disk M dwarfs differ significantly in metallicity. Multi-color deep CCD surveys are uniquely capable of detecting these separate populations of stars with differing metallicity and therefore colors. Analyzing very deep threeband CCD images covering $192 \operatorname{arcmin}^{2}$ at high galactic latitude we find no evidence for a population of extreme low mass $M$ subdwarfs sufficient to account for the halo dark matter. These observations covering a volume of $2 \times 10^{5} \mathrm{pc}^{3}$ are consistent with extrapolations of a halo luminosity function determined using low metallicity stars in the solar neighborhood.
\end{abstract}

\section{INTRODUCTION}

With the recent discovery of microlensing events by objects in the halo of our galaxy (Alcock et al. 1993), there has been a resurgence of interest in examining low mass stellar objects as the source of an important fraction of the unseen matter in our Galaxy. While direct constraints on the stellar mass function may be obtained by microlensing, a significant excess of low mass objects with $M \leq 0.1 M_{\odot}$ would have observable consequences for the faint tail of the stellar luminosity function (LF). Our knowledge of the shape of the stellar LF has been limited until recently to the local neighborhood within $10-20 \mathrm{pc}$ of the sun, and covers a mixture of population types.

The recent results for the halo $M$ subdwarf population in the solar neighborhood by Dahn et al. (1994) using measured trigonometric parallaxes are generally consistent with the earlier results of Dawson (1986) based on a statistical analysis of proper motions. Both of these studies suggest a flat or falling $\mathrm{LF}$ at the faint end where $M_{V}=14-15$, close to the theoretical estimate for the hydrogen burning limit for stars with halo metallicities (Monet et al. 1992). This appears to be at odds with one recent survey for halo stars; deep V, R and I-band CCD observations were used by Richer and Fahlman (1992) to estimate photometric parallaxes of halo stars more than $1200 \mathrm{pc}$ above the galactic plane. In contrast to the solar neighborhood results, Richer and Fahlman's analysis suggests that the faint end halo LF and mass functions are steeply rising. 


\section{The Results of Deep CCD Field Surveys}

\section{OBSERVATIONS}

Very deep multicolor CCD surveys provide a rich database which can be exploited to sample the low mass stellar halo population out to several $\mathrm{kpc}$, provided that proper color calibration is available to separate low mass stars of differing metallicities as well as morphologically misclassified galaxies. Deep three-band ( $\left.B_{J}<27.5, R<26.4, I<25 \mathrm{mag}\right)$ CCD images of 12 widely spaced high galactic latitude fields covering a total of $144 \operatorname{arcmin}^{2}$ on the sky have been obtained as part of a 4-m survey done at CTIO originally to study the faint galaxy count problem. Together with a single $1024^{2} \mathrm{CCD}$ field covering 48 $\operatorname{arcmin}^{2}$ on the sky obtained at KPNO, these data covering $192 \operatorname{arcmin}^{2}$ have been analyzed to search for $\mathrm{M}$ dwarfs near the halo and disk hydrogen burning limits. A detailed discussion of the observations, image processing, and stargalaxy separation techniques can be found in Boeshaar et al. (1986) and Tyson (1988).

Our color data have been carefully calibrated by observing standard stars of different temperatures and luminosities which have spectroscopically determined metallicities. Many of these observations were necessary in order to show how to separate out the different population types in our color-color plane (Gullixon et al. 1995). Metal deficient halo $M$ stars have redder $B_{J}-R$ colors than their disk counterparts due to decreased molecular band blanketing that dominates the $\mathrm{V}$ through I passbands. As can be seen in Figure 1, the $\mathrm{M}$ subdwarfs of varying metal deficiency lie above their disk population counterparts in our color-color plane, with the objects of lowest metallicity having the reddest colors in $B_{J}-R$. The M subdwarfs in this diagram have been chosen from the sample of Dahn et al. (1994), and the object with the reddest R-I color in Figure 1 is LHS 1742a, one of the least luminous and most metal poor M subdwarf discovered to date. The extreme halo population $M$ stars plotted cover $M_{V} \approx 13-14.5$. The disk $M$ dwarfs encompass the complete spectral range $\mathrm{dM} 0$ - dM9, or $M_{V} \approx 8-19.5$.

A comparison with color-color data for galaxies found in our fields, most of which are very blue, indicates that no galaxies are found redward of $R-I=3$. This is the region where the latest $\mathrm{M}$ dwarfs are found. Furthermore, minimal galaxy contamination is expected in the region where the halo population $\mathrm{M}$ subdwarfs are located.

\section{RESULTS}

If LHS 1742a is representative of the least luminous halo $M$ subdwarfs near the hydrogen burning limit, then we might expect such objects to have $M_{V} \approx 15$. At this absolute magnitude, our survey is complete out to $3000 \mathrm{pc}$, covering a volume of approximately $205,000 \mathrm{pc}^{3}$. There are six objects in our deep CCD survey data having colors consistent with $\mathrm{M}$ subdwarfs of $M_{V} \approx 13.5-15$. A comparative analysis of our galaxy data in the same fields at similar apparent magnitudes suggests that this subdwarf sample may be contaminated by at most 1-2 misclassified compact high redshift galaxies of similar color which appear stellar. However pollution of our ground-based stellar counts by misclassified very red galaxies is not a major problem. There appear to be few very red galaxies which are faint and compact: recent HST WFPC2 Medium Deep Survey data reveal no new population of faint red compact galaxies (Griffiths et al. 1994). 


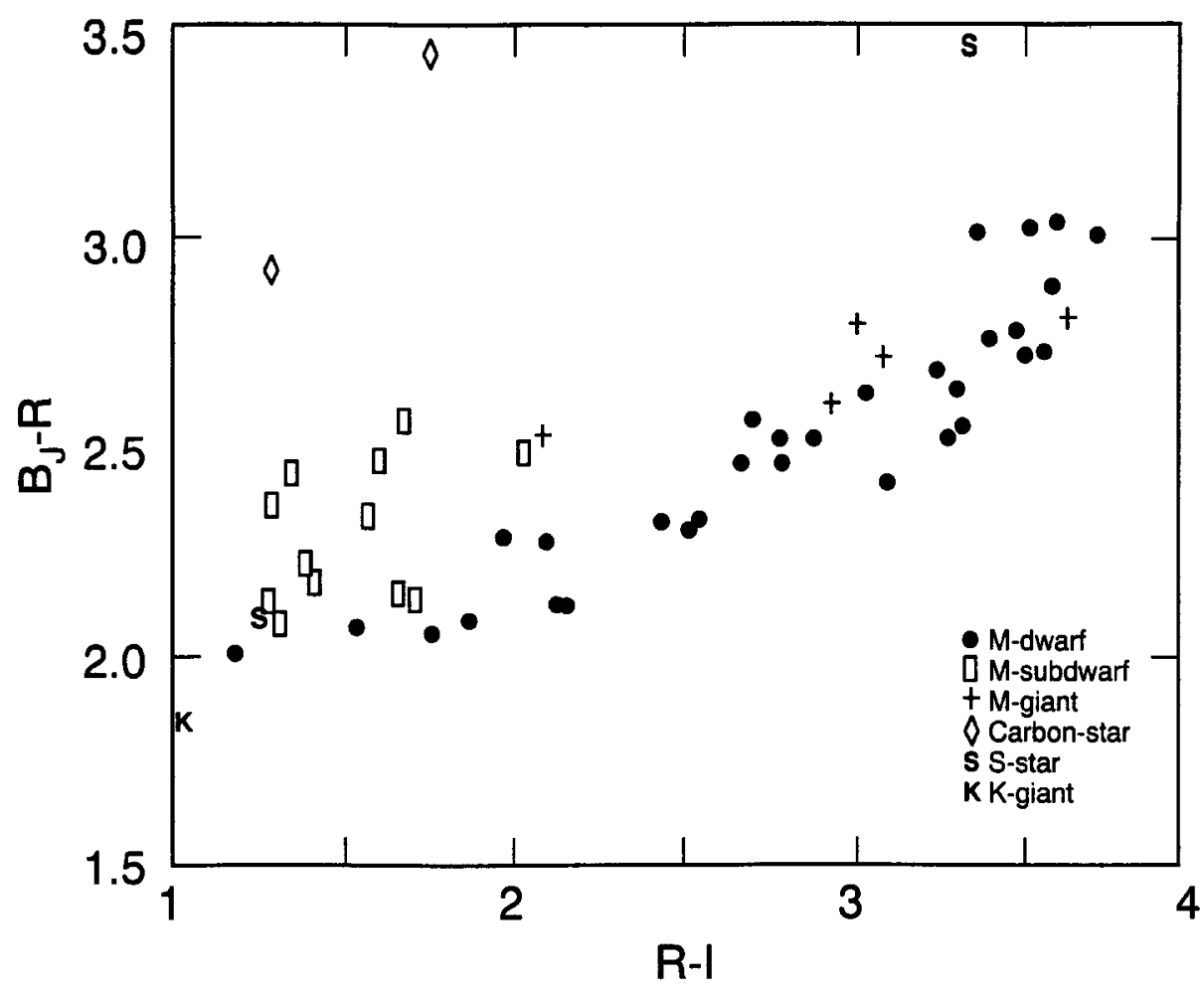

Figure 1.Colors of red standard stars with spectral classification observed on the $B_{J}, R, I$ system. Disk $M$ dwarfs cover the complete spectral range dMO$d M 9$, while the halo $M$ subdwarfs have been selected from the list of Dahn et al. (1994), representative of the complete observed range in metal deficiency and temperature.

Our subdwarf counts agree quite well with the predictions made using the luminosity function of Dahn et al. (1994), i.e. that we should find $5 \pm 3$ of the least luminous subdwarfs within our volume. By comparison, the halo luminosity function of Richer and Fahlman (1992) would predict over five times as many low mass $M$ subdwarfs than we detect in our surveys. This is clearly not the case, even though we go at least as deep their survey. If the dark matter halo were composed of metal poor halo stars near the hydrogen burning limit with color similar to the reddest subdwarfs observed in Figure 1, then we should have detected over 2000 such objects. Our data also eliminates the possibility that $0.092 M_{\odot}$ zero metallicity stars at the hydrogen burning limit as modeled by Saumon et al. (1994) constitute a major portion of the halo dark matter. Such objects would be found approximately $1 \mathrm{mag}$ bluer in R-I and $0.5 \mathrm{mag}$ bluer in $B_{J}-R$ than our reddest halo population stars, a region with three or four objects. We suspect that many of these latter objects are misclassified galaxies.

In time, parallel HST WFC2 very deep multicolor imaging should improve on these results because of its superior angular resolution. While multicolor data is crucial for studying these dwarf stellar populations, Bahcall et al. (1994) 
recently reported HST observations in one color of a single $4.4 \mathrm{arcmin}^{2}$ field, in F606W and F814W bands. Using their I limit of $25.3 \mathrm{mag}$ and an $M_{I}$ of 11.5 (Monet et al. 1992) as representative of the latest halo extreme M subdwarfs identified spectroscopically, we estimate that Bahcall et al. have surveyed a volume one fifth of that for which we have accurate deep CCD color data. Two objects in their data have $V-I \leq 3$ colors and distances consistent with the $M$ subdwarfs defined above, i.e. results statistically comparable to ours. In their interpretation they assume that that the least luminous halo and disk stars have similar V-I colors. However, it is known that faint halo and disk stars differ in color. It is not surprising that Bahcall et al. discovered no stars with V-I greater than 3, if such objects belong to the disk population as current theory (Saumon et al. 1994, and references therein) suggests. Assuming an average disk M dwarf scale height of $350 \mathrm{pc}$, Bahcall et al. sampled a few tens of cubic parsecs for disk dwarfs. Using the best current measurements of the disk $\mathbf{M}$ dwarf luminosity function (Kirkpatrick et al. 1994), at most one $V-I>3$ and no V-I $=4-5$ disk $\mathrm{M}$ dwarfs would be expected in that HST field.

Recent evidence (Kirkpatrick et al. 1994) suggests that the latest $M$ dwarfs with V-I's of 4-5 may have a smaller scale height than earlier disk M dwarfs and that their distribution is nonuniform. Our survey has detected six stellar objects with colors indicative of $\mathrm{M}$ dwarfs having $V-I \approx 4-5$ and $M_{I} \approx 14$ $\left(M_{V} \approx 19\right)$, all located in our single $1024^{2}$ field. Assuming these objects are among the latest $M$ dwarfs, their I magnitudes indicate that they all lie within $250 \mathrm{pc}$ of the galactic plane, though our survey is complete to $300 \mathrm{pc}$ for such objects.

\section{REFERENCES}

Alcock, C. et al. 1993, Nature, 365, 621

Bahcall, J., Flynn, C., Gould, A., \& Kirhakos, S. 1994, ApJ 435, L51

Boeshaar, P.C., Tyson, J.A., Seitzer, P. 1986, in Astrophysics of Brown Dwarfs, eds. Kafatos, M.C., Harrington, R.S., Maran, S.P. (Cambridge U. Press, NY), p. 76

Dawson, P.C. 1986, ApJ 311, 984

Dahn, C.C. and Liebert, J., Harris, H.C., and Guetter, H.H. 1994, in Proceedings of the ESO Workshop "The Bottom of the Main Sequence And Beyond", ed. C.G. Tinney (Springer-Verlag, Heidelberg), in press

Griffiths, R.E. et al. 1994, ApJ Lett., 435, L19

Gullixon, C.A., Boeshaar, P.C., Tyson, J.A., Seitzer, P. 1995, ApJS, in press

Kirkpatrick, D. et al. 1994, ApJS 94, 749

Monet, D.G., Dahn, C.C., Vrba, F.J., Harris, H.C., Pjer, J.R., Luginbuhl, C.B., Ables, H.D. 1992, AJ 103, 638

Richer, H.B., and Fahlman, G.G. 1992, Nature 358, 383

Saumon, D., Bergeron, P., Lumine, J.I., Hubbard, W.B., Burrows, A. 1994, ApJ 424,333

Tyson, J.A. 1988, AJ 96, 1 